\title{
ROS-induced endothelial stress contributes to pulmonary fibrosis through pericytes and Wnt signaling
}

\author{
Annika Andersson-Sjöland, Jenny C Karlsson and Kristina Rydell-Törmänen
}

Pulmonary fibrosis is a grave diagnosis with insidious progression, generally considered as a consequence of aberrant epithelial wound healing and excessive scarring. This process is commonly modeled in animals by local bleomycin administration, resulting in peribronchial inflammation and subsequent fibrosis. We have previously described initiation and early development of distal pulmonary fibrosis following repeated subcutaneous bleomycin injections (systemic administration). The aim of this study was to identify mechanisms for the development of pulmonary fibrosis, which we hypothesize is related to endothelial stress and activation. Bleomycin was administered subcutaneously 3 times/ week during $0.33-4 \mathrm{w}$, and parenchymal alterations were studied. In addition, we used microvascular endothelial cells to investigate effects of bleomycin in vitro. Our results confirmed that systemic administration of bleomycin exerts oxidative stress indicated by an increase in Sod1 at $0.33,1$, and $4 w(P<0.05)$. Endothelial cells were activated (increased CD106 expression) from $1 \mathrm{w}$ and onwards $(P<0.05)$, and p21 expression was increased $2-3$ times throughout the study $(P<0.05)$ as were the number of $\beta$-catenin-positive nuclei $(P<0.001)$. Wnt3a was increased at $0.33,1$, and $4 \mathrm{w}(P<0.01)$ and Wnt5a from $1 \mathrm{w}$ and onwards $(P<0.001)$. The present study suggests that bleomycin-induced reactive oxygen species (ROS) causes DNA stress affecting the endothelial niche, initiating repair processes including Wnt signaling. The repeated systemic administrations disrupt a normally fine-tuned balance in the Wnt signaling. In addition, pericyte differentiation was affected, which may have significant effects on fibrosis due to their ability to differentiate into myofibroblasts. We conclude that the endothelial niche may have an important role in the development of pulmonary fibrosis and warrants further investigations.

Laboratory Investigation (2016) 96, 206-217; doi:10.1038/labinvest.2015.100; published online 14 September 2015

Pulmonary fibrosis is a grave and debilitating diagnosis of unknown or inadequately known pathology. The insidious progression of clinical pulmonary fibrosis has seriously hampered the understanding of the pathological development and mechanisms, but it is now generally recognized that an aberrant epithelial wound healing in association with dysregulated fibroblast proliferation and matrix synthesis has a key role in the disease development. ${ }^{1}$

To investigate the initiation and early development of pulmonary fibrosis animal models are commonly used, one of the most common being local bleomycin administration. Local (intratracheal) bleomycin administration causes epithelial necrosis and severe inflammation severely affecting all cells within the central part of the lung, which is followed by development of a heterogeneous fibrosis and later resolution. Bleomycin exerts it's effect through generation of reactive oxygen species (ROS), which results in oxidative stress in lung also after systemic administration. ${ }^{2,3}$ In addition, the oxidative effects of bleomycin in vitro have also been well described. ${ }^{4,5}$ In contrast to intratracheal administration of bleomycin, repeated subcutaneous administrations result in a systemic exposure, and cause distal fibrosis that develops in parallel with mild inflammation. ${ }^{6}$ The mechanisms behind these events are unknown, although it is likely that the endothelial niche is involved as previous studies have described systemic bleomycin administration (albeit at a higher dose) to result in capillary damage. ${ }^{7}$

The endothelial niche consists of endothelial cells and pericytes, which are vascular-associated progenitors. Pericytes were originally defined by their location within the basement membrane of vessels, spanning multiple endothelial cells, and for being crucial for endothelial integrity. Loss of these progenitors has been associated with endothelial dysfunction. ${ }^{8}$ Recently, pericytes have been suggested to be a type of mesenchymal stem cells (MSCs) due to their capacity to differentiate into osteoblasts, chondrocytes, and adipocytes. ${ }^{9}$

Unit of Lung Biology, Department of Experimental Medical Science, Lund University, Lund, Sweden

Correspondence: Dr K Rydell-Törmänen, PhD, Unit of Lung Biology, Department of Experimental Medical Science, Lund University, BMC C12/Klinikgatan 30, Lund S221-84, Sweden.

E-mail: Kristina.Rydell-Tormanen@med.lu.se

Received 13 January 2015; accepted 5 May 2015 
An important regulator of stem cell homeostasis is the Wingless/Integrase-1 (Wnt)-signaling pathways, ${ }^{10}$ which are believed to keep stem cells in a dedifferentiated and selfrenewal state. ${ }^{11,12}$ Wnt signaling is an evolutionary conserved signal transduction cascade, transmitting signals through cell-cell contact and regulates cell fate, polarity, differentiation, migration and thereby organogenesis and fetal development. ${ }^{13}$ Small RhoGTPases represent key mediators for facilitating Wnt-induced intracellular signaling, promoting transcriptional as well as morphological changes in cell behavior. ${ }^{14}$ Wnt signaling is essential during fetal lung development, ${ }^{15}$ but is also involved in tissue repair ${ }^{16}$ and stem cell homeostasis ${ }^{10}$ in adults. In addition, several studies have shown the importance of Wnt signaling in pulmonary fibrosis. ${ }^{17,18}$

The aim of the present study was to identify a mechanism for the development of pulmonary fibrosis following subcutaneous bleomycin administration. For greater accuracy, we used histology enabling us to specifically investigate the lung parenchyma.

Our results suggest that bleomycin induces endothelial stress and repeated exposures disrupt a normally fine-tuned Wnt balance, causing MCS-like pericytes to alter phenotype. ${ }^{19}$ We hypothesize that these involvements ultimately lead to pulmonary fibrosis, likely by differentiation of pericytes into myofibroblasts, as recently described. ${ }^{20}$ As pericytes have the ability to differentiate into myofibroblasts as well as to exert regulatory influences on adjacent cells, understanding the effects of Wnt signaling in pericytes, now emerges as an important objective to understand the early events of pulmonary fibrosis.

Some of the results of this study have previously been reported in the form of an abstract. ${ }^{19}$

\section{MATERIALS AND METHODS Model}

We used a previously described model where subcutaneous bleomycin injections were used to initiate pulmonary fibrosis. ${ }^{6}$ Briefly, $0.1 \mathrm{ml}$ bleomycin (500 IE/ml, Bleomycinsulphate, Bleomycin Baxter, Baxter Medical AC, Kista, Sweden) was administered (using a 30 gauge needle) subcutaneously on the back, 3 times/week for up to 4 weeks, and animals were killed $24 \mathrm{~h}$ after final injection. Controls received saline injections. Animals were kept housed under barrier conditions, with food and water ad libitum. All protocols were approved by the local ethics committee (Malmö/Lund, Sweden; number M275-10). Following killing, tissues were fixed in in $4 \%$ formaldehyde, dehydrated, and embedded in paraffin.

\section{Immunohistochemistry}

In all, $4.5 \mu \mathrm{m}$ sections were processed for immunohistochemistry (IHC) according to standard protocol. Briefly, sections were rehydrated and allowed to equilibrate in TBS buffer for $15 \mathrm{~min}$. The sections were pretreated with Proteinase $\mathrm{K}$ solution $(20 \mathrm{mg} / \mathrm{ml})$ in $37^{\circ} \mathrm{C}$ for $30 \mathrm{~min}$. The primary antibody was applied, diluted in Dilution buffer (TBS supplemented with $1 \%$ BSA) and incubated in room temperature for $1.5-2 \mathrm{~h}$ (room temperature) or overnight $\left(4{ }^{\circ} \mathrm{C}\right)$. Following incubation with primary antibody, the slides were rinsed and secondary antibody+nuclear marker DAPI was applied. The dilution of the secondary antibody used was 1:200. DAPI was used at a concentration of $30 \mu \mathrm{M}$ and further diluted 1:200 when mixed with the secondary antibody. The mixture of secondary antibody and DAPI was incubated for $45 \mathrm{~min}-1 \mathrm{~h}$ in room temperature before rinsing. The slides were mounted in fluorescent mounting medium and stored in $-20{ }^{\circ} \mathrm{C}$ before analysis. The following antibodies were used within this study: Sod1 (1:300, ab13498, Abcam, Cambridge, UK), p21 (1:200, ab7960, Abcam), CD106/VCAM-1 (1:200, ab19569, Abcam), NG2 (1:100, MAB5384A4, EMD Millipore Corporation, Billerica, MA, USA), Wnt3a (1:300, 09-162, EMD Millipore), Wnt5a (1:100, ab72583, Abcam), $\beta$-catenin (1:100, AF1329, R\&D Systems, Minneapolis, MN, USA), CD31 (1:100, ab56299, Abcam), Vimentin (1:600, ab92547, Abcam), CDC42 (1:500, ab64533, Abcam), panRhoRacCDC42 (1:300, ab69091, Abcam), and $\alpha$-SMA (1:2500, C6198, Sigma, St Louis, MO, USA).

\section{Histological Analysis}

All analyses were performed by digital imaging and the software ImageJ (v1.44j; Wayne Rasband, NIH, USA) was used. All slides were analyzed in a blinded manner where images of the lung parenchyma were obtained randomly. To be able to compare between slides, a starting point at the bottom part of the section close to (directly under) the pleura was selected. From this starting point, the next image was taken 2-4 image fields right and 1-3 upwards from the starting image. A variable number of images of each slide were taken, ranging from 4 to 10, depending on the magnification and staining. Analysis of the images was performed slightly different depending on the parameter studied:

(1) The positively labeled area was measured using a threshold to define positive staining. This area was related to the total tissue area, thus giving the results as fraction of positive staining of total tissue area (\%).

(2) The number of positively labeled cells (or nuclei, in the case of $\beta$-catenin) was counted and related to the tissue area, thus results are given as number of positively labeled cells (nuclei)/area.

\section{Transmission Electron Microscopy}

To investigate the integrity of the vascular wall, we used transmission electron microscopy (TEM) and studied samples from control animals and animals subjected to $2 \mathrm{w}$ of bleomycin. Within the samples, vascular walls of capillaries were investigated for signs of endothelial dysfunction, and we sought to identify pericytes within the vascular wall to determine their presence. 


\section{Microvascular Endothelial Cells}

Primary lung microvascular endothelial cells (from C57/bl6 mice, product number C57-6011) were purchased (CellBiologics, Chicago, IL, USA) and grown according to instructions provided by the supplier in complete mouse endothelial cell culture medium (M1168, CellBiologics).

\section{Bleomycin Administration In Vitro}

For experiments, cells were plated in gelatinase-coated 12-well plates and grown to confluency, according to the manufacturer's instructions. Before bleomycin administration, medium was changed and bleomycin (Baxter, $1 \mathrm{IE} / \mathrm{ml}$ in $\mathrm{NaCl}$ ) was added to a final concentration in medium of $0.3 \mathrm{IE} / \mathrm{ml}$. The experiment was performed twice and data are given as mean of the two experiments. Cells were harvested after $24 \mathrm{~h}$ for extraction of total RNA using RNeasy (Qiagen GmBH, Hilden, Germany) according to the manufacturer's instructions (Qiagen).

\section{RT-qPCR}

The quantity of RNA was measured by spectrophotometry using a NanoDrop 2000c (Thermo Fisher Scientific, Gothenburg, Sweden). Total RNA $(1 \mu \mathrm{g})$ was reverse-transcribed using superscript II according to the manufacturer's manual (Invitrogen, Carlsbad, CA, USA) and stored at $-70^{\circ} \mathrm{C}$. In all, $5 \mu \mathrm{l}$ cDNA (diluted 1:250) was mixed with $15 \mu \mathrm{l}$ SYBR-green PCR master mix (Applied Biosystems, Life Technologies) and amplified by RT-qPCR using Agilent Technologies (Santa Clara, CA, USA). The samples were held for $10 \mathrm{~min}$ at $95^{\circ} \mathrm{C}$, before being cycled for 40 cycles of $30 \mathrm{~s}$ at $95^{\circ} \mathrm{C}, 1 \mathrm{~min}$ at $59^{\circ} \mathrm{C}$, and $1 \mathrm{~min}$ at $72^{\circ} \mathrm{C}$. Each sample was analyzed in triplicate. Reactions were performed using MX3000P 96-well plates (Agilent Technologies). Primers detecting Sod-1 and p21 (PrimerDesign, Southampton, UK), and RhoA and Racl (Eurofins GmbH, Ebersberg, Germany) were used:

Sod1

S: TTGGGCAAAGGTGGAAATGAA, AS: ACTCAGACCA CACAGGGAAT

$$
\text { p21 }
$$

S: TGACTCCAGCCCCAAACAT, AS: GGGAGGGAGCCA CAATACA

\section{RhoA}

S: ACAACTGCATCCCAGAACCT, AS: TACCACAAGCTC CATCACCA

Racl

S: TATGGGACACAGCTGGACAA, AS: GGACTCACAAG GGAAAAGCA.

\section{Statistical Analysis}

Results were statistically tested using Analyse-it for Microsoft Excel (Analyse-it Software, Ltd, Leeds UK). For determination of statistical differences between the different groups the Kruskal-Wallis combined with the LSD post hoc test was performed, and all groups were compared pairwise. $P<0.05$ was considered as statistically significant, and data are, if not stated otherwise, given as mean \pm s.d.

\section{RESULTS}

Representative images of all IHC stainings, at all time points including negative controls can be found in the Supplement (Supplementary Figure 1).

\section{Confirmation of Oxidative Stress in Lung}

To confirm that bleomycin exert oxidative stress on lung tissue, we investigated the expression of the ROS-neutralizing enzyme Superoxide dismutase-1 (Sod1) and a marker of cell stress, p21, in lung.

Significantly increased expression of Sod1 (Figure 1a and c) was found at $0.33,1 \mathrm{w}$, and $4 \mathrm{w}(0.15 \pm 0.06 \%, 0.19 \pm 0.09 \%$, and $0.19 \pm 0.06 \%$ ), compared with controls $0.08 \pm 0.04 \%$ $(P=0.0288,0.0058$, and 0.003 , respectively $)$. At 2 and 3 weeks, the expression of Sod 1 was normalized.

The number of p21-positive cells (Figure 1d and g) was increased 2-3 times after one dose of bleomycin $(P=0.0004-$ $0.0197)$, compared with controls $\left(85 \pm 47\right.$ cells $\left./ \mathrm{mm}^{2}\right)$, and remained increased throughout the study.

Administration of bleomycin to microvascular endothelial cells in vitro showed no increased mRNA expression of Sod1 (Figure 2a), whereas expression of p21 was increased 2.5-3.7 times (Figure $2 b$ ).

\section{Bleomycin Induces Endothelial Stress}

We wanted to investigate the effect of bleomycin in the endothelial niche and we therefore investigated the expression of CD106 (VCAM-1), which is a hallmark of activated endothelial cells. Our results showed that CD106 increased slowly (Figure $3 \mathrm{a}$ and $\mathrm{c}$ ), and was significantly increased at $1 \mathrm{w}(0.15 \pm 0.08 \%$ of tissue area), $2 \mathrm{w}(0.29 \pm 0.13 \%), 3 \mathrm{w}$ $(0.24 \pm 0.1 \%), \quad$ and $4 \mathrm{w} \quad(0.26 \pm 0.06 \%)$ compared with controls $(0.13 \pm 0.07 \%)$. Interestingly, the variation was relatively large, but decreased over time, suggesting a variable perfusion of the lung and thus exposure to bleomycin.

The increased expression of CD106 confirmed an effect of bleomycin on the endothelial niche, and we therefore wanted to investigate the pericytes within the niche by staining for NG2 (Figure 3d and f). Our results showed that the number of NG2-positive cells decreased rapidly after one bleomycin administration, and remained decreased at 1 and $2 \mathrm{w}(43 \pm 13$ and $55 \pm 13$ cells $/ \mathrm{mm}^{2}$, respectively) compared with controls $\left(75 \pm 12\right.$ cells $\left./ \mathrm{mm}^{2}\right)$. The numbers were normalized at $3 \mathrm{w}$, but at $4 \mathrm{w}$ the number of NG2-positive cells were again decreased $\left(46 \pm 13\right.$ cells $\left./ \mathrm{mm}^{2}\right)$. Importantly, we found no obvious signs of endothelial dysfunction (e.g., significant edema or endothelial apoptosis/necrosis). We noted however that the intensity of the NG2 staining appeared to be lower after bleomycin, ie, positive cells appeared less bright.

Double stainings for CD106/ $\alpha$-SMA (Figure 3g) and NG2/ $\alpha$-SMA (Figure $3 \mathrm{~h}$ ) were made to investigate whether activated endothelial cells or pericytes expressed $\alpha$-SMA. 

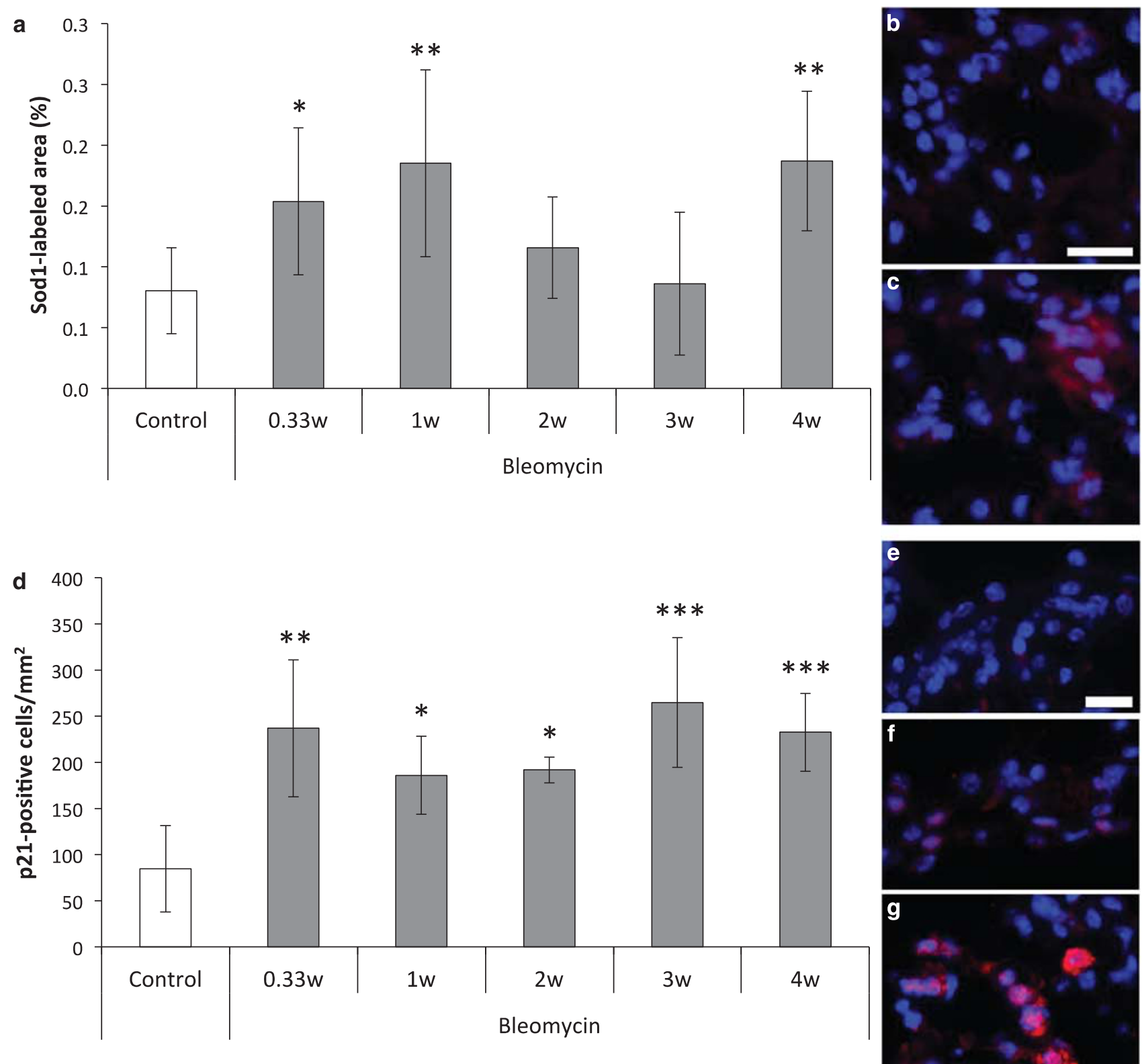

Figure 1 Subcutaneous bleomycin generates ROS and induces DNA stress in lung. To confirm oxidative stress in lungs following bleomycin administrations, Superoxide dismutase (Sod)-1 was investigated (a). Sod1 (red staining) increases rapidly after the first bleomycin injection (0.33w), compared with controls (b). At 2 and $3 w$, the levels are normalized, but at $4 w$ (c) a significant increase is again present. Cellular effects of the bleomycin-induced ROS were confirmed by staining with p21 (d), which is expressed within the nucleus (blue) in response to DNA stress. In similarity to Sod1, p21 (red staining) also increases rapidly, after one injection (f, $0.33 \mathrm{w})$ compared with controls (e), but remains increased throughout the study, shown here at $4 \mathrm{w}(\mathbf{g})$. Nuclear localization of p21 can be clearly seen as purple staining. Data are given as either fraction (\%) labeled area of total tissue area (a) or number of positively labeled nuclei $/ \mathrm{mm}^{2}$ (d), and given as mean \pm s.d. Scale bars represent $20 \mu \mathrm{m}$ in all images. Bleomycin-treated groups were compared with controls using the Kruskal-Wallis test combined with LSD post hoc test, ${ }^{*} P<0.05,{ }^{* *} P<0.01$, and ${ }^{* * *} P<0.001$. LSD, least significant difference; ROS, reactive oxygen species.

The results suggest expression of actin in some endothelial cells (Figure 3g, arrowheads) and potentially also pericytes (Figure 3g, arrow), although little co-positivity of CD106 and $\alpha$-SMA was found. Some scattered cells co-positive for NG2 and $\alpha$-SMA were found (Figure $3 \mathrm{~h}$, arrowhead), in addition to cells positive only for NG2 (Figure $3 \mathrm{~h}$, arrow).
To further study the integrity of the endothelial cell layer we used TEM, and found the endothelial cell layer to be structurally similar after $2 \mathrm{w}$ of bleomycin compared with control (Figure 4). We were able to identify pericytes in both controls and bleomycin $2 \mathrm{w}$, with very similar morphology, and in this high magnification we were also able to detect a 
slight edema within the vascular wall after bleomycin administration.

\section{Effects on Wnt3a and Wnt5a}

Wnt3a increased rapidly (Figure 5a), after one injection $\left(132 \pm 28\right.$ cells $\left./ \mathrm{mm}^{2}\right)$ compared with controls $(45 \pm 10$ cells/ $\mathrm{mm}^{2}$ ), but decreased rapidly again. The expression was normal at 2 and $3 \mathrm{w}$. However, at $4 \mathrm{w}$ the number of Wnt3a-positive cells was again significantly increased $\left(155 \pm 21\right.$ cells $\left./ \mathrm{mm}^{2}\right)$.

Wnt5a also increased after bleomycin, but responded more slowly and was significantly increased at $1 \mathrm{w}(0.2 \pm 0.1 \%$ of tissue area) compared with controls $(0.07 \pm 0.05 \%$ of tissue area $)$ and onward, peaking at $4 \mathrm{w}(2.3 \pm 0.9 \%$ of tissue area, Figure $5 \mathrm{~b})$.

Wnt3a is able to initiate canonical (Wnt/ $\beta$-catenin) signaling, but is in itself not a marker for this signaling cascade. We therefore investigated the number of cells where $\beta$-catenin was relocalized to the nucleus, which is a hallmark of ongoing canonical signaling. Our results showed that the number of $\beta$-catenin-positive nuclei was increased at all times compared with controls $(P<0.001$ for all $)$, and peaked at $4 \mathrm{w}$ when $271 \pm 69$ positively stained nuclei/ $\mathrm{mm}^{2}$ were found compared with $36 \pm 10$ in controls (Figure $5 c$ ).

The expression patterns of both Wnt 3 a and Wnt5a along with $\beta$-catenin suggest their expression to be connected, but temporally shifted with Wnt3a and $\beta$-catenin responding more rapidly to tissue stress, whereas Wnt5a reacted slower (Figure $5 \mathrm{~d}$ ). At $3 \mathrm{w}$ both Wnt $3 \mathrm{a}$ and Wnt5a decreased slightly, whereas $\beta$-catenin showed no such trend. All markers of Wnt signaling peaked at $4 \mathrm{w}$.

Furthermore, double stainings were performed for Wnt3a $+\mathrm{NG} 2$, Wnt5a+NG2, and Wnt3a+Wnt5a to investigate co-localization of these markers (Figure 6). The images
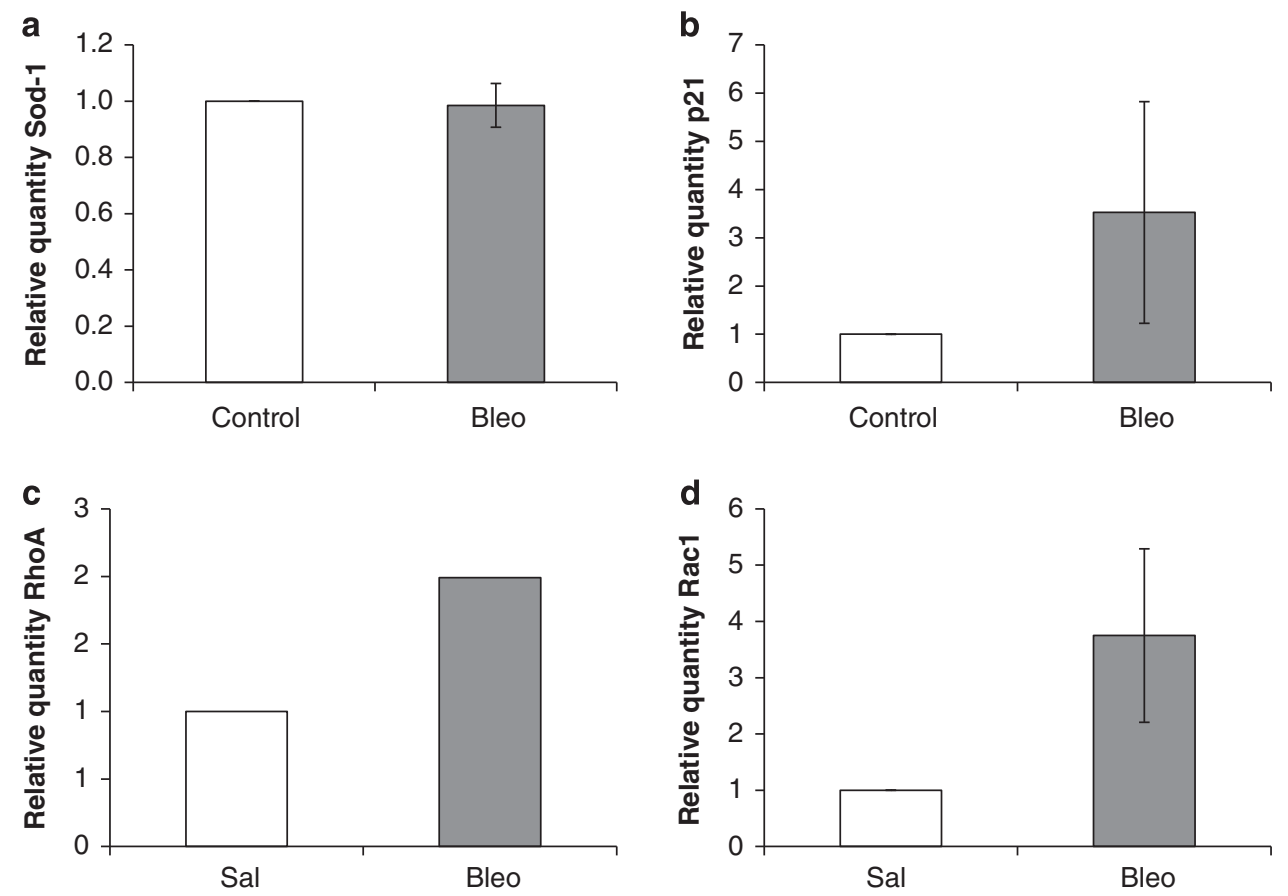

Figure 2 Bleomycin exerts effects on microvascular endothelial cells in vitro. To investigate the reaction to bleomycin exposure on endothelial cells, lung microvascular endothelial cells were exposed to bleomycin in vitro (confluent cells were exposed to bleomycin $0.3 \mathrm{IE} / \mathrm{ml}$, for $24 \mathrm{~h}$ ) and the reaction analyzed by RT-qPCR. Our results failed to show any effect of bleomycin on Sod1 transcription (a), whereas bleomycin increased expression of p21 2.5-3.7 times (b), RhoA 2x (c), and Rac1 2.7-4.8 times (d). The experiment was repeated twice, and results are given as mean of these two experiments.

Figure 3 Bleomycin affects the endothelial niche. To determine whether the oxidative stress affects the endothelial cells, we visualized CD106 (VCAM1, green staining), which is expressed on activated endothelial cells (a). In comparison with controls (b) we found endothelial cells to be activated at 1,2 (c), 3 and $4 \mathrm{w}$, whereas no increases were found at $0.33 \mathrm{w}$. The other cell type of the endothelial niche is pericytes, and we therefore investigated the number of pericytes using NG2 as a marker (d). Surprisingly, compared with controls (e) we found the number of NG2-positive cells (green staining) to decrease rapidly following bleomycin administrations; and were significantly decreased at 0.33 (f), 1, 2, and 4w. Double stainings for CD106/a-SMA (g) and NG2/a-SMA (h) were made to investigate whether activated endothelial cells or pericytes expressed $a$-SMA. The results suggest expression of actin in some endothelial cells ( $\mathbf{g}$, arrowheads) and possibly also pericytes ( $\mathbf{g}$, arrow), although no co-positivity of CD106 and $\alpha$-SMA was found. Few cells were found to be co-positive for NG2 and $a$-SMA (h, arrowhead), in addition to cells positive only for NG2 (h, arrow). Scale bars represent $20 \mu \mathrm{m}$ in all images, and vessels are indicated by $v$. Data are given as either fraction (\%) labeled area of total tissue area (a) or number of positively labeled cells/ $\mathrm{mm}^{2}(\mathbf{d})$, and given as mean \pm s.d. Bleomycin-treated groups were compared with controls using the Kruskal-Wallis test combined with LSD post hoc test, ${ }^{*} P<0.05,{ }^{* *} P<0.01$, and ${ }^{* * *} P<0.001$. LSD, least significant difference. 
showed that pericytes were weakly positive for Wnt3a in healthy lung (Figure $6 \mathrm{a})$, but after one injection $(0.33 \mathrm{w})$ the number of pericytes was decreased and Wnt3a expression was increased (Figure 6b). We found Wnt5a expression at low levels, but seldom in pericytes, in healthy lung (Figure 6c). In contrast, after $4 \mathrm{w}$ of bleomycin, total Wnt5a expression increased and some Wnt5a-positive pericytes could be seen (Figure 6d). Double staining for Wnt3a and Wnt5a showed both double-positive cells and Wnt3a single-positive cells. Very few Wnt5a single-positive cells were found in normal
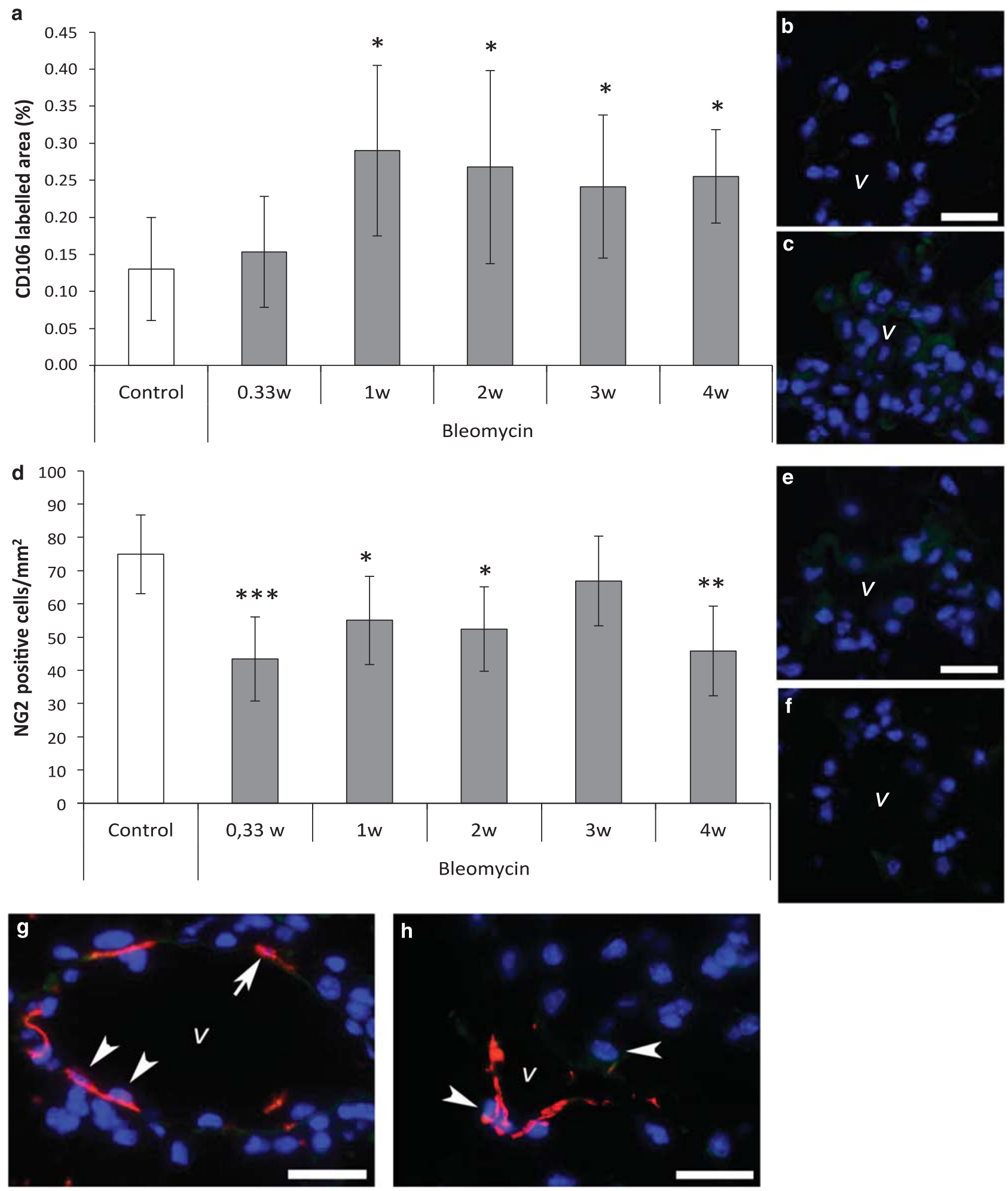
lung (Figure 6e). Following one bleomycin administration, the expression of Wnt3a was increased, and the cells rarely expressed Wnt5a (Figure 6f). After 4w of bleomycin however, several cells co-positive for Wnt5a and Wnt3a were found (Figure 6g).

Recently, a study described a switch from canonical to noncanonical signaling in hematopoietic stem cells (HSCs), and the small RhoGTPase CDC 42 mediated the transition. ${ }^{21}$ We therefore investigated tissue expression of $\mathrm{CDC} 42$ in
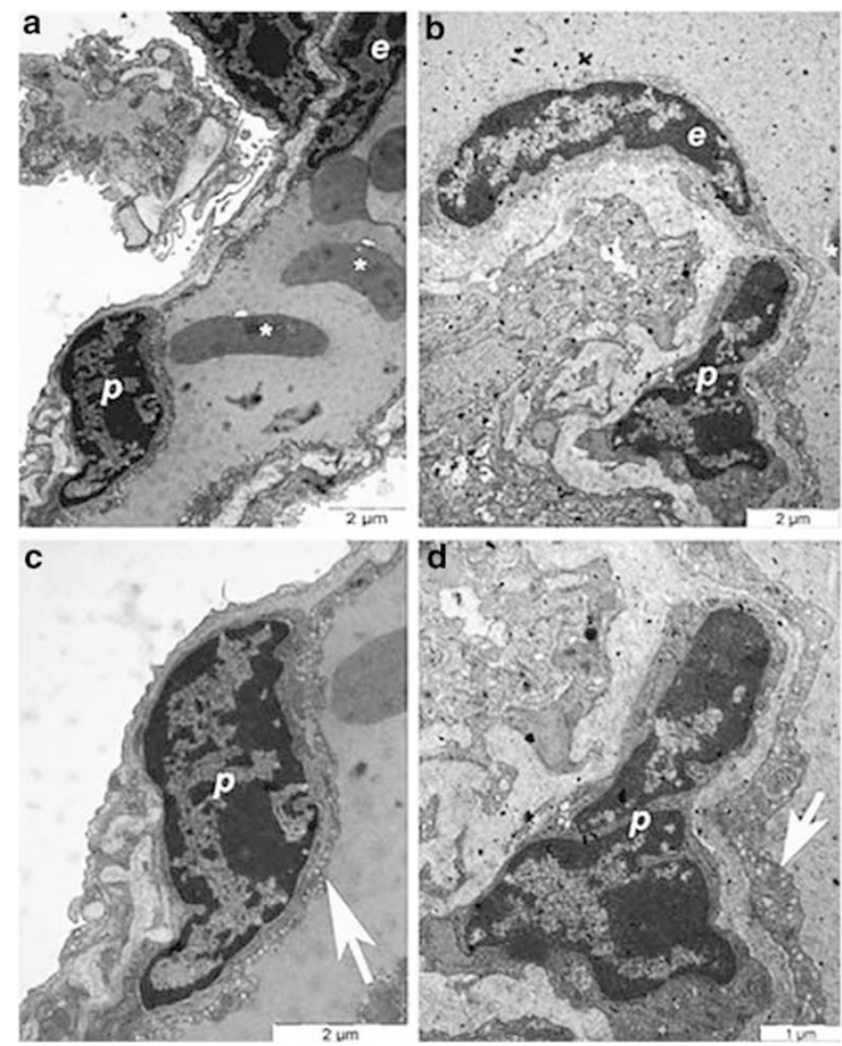

Figure 4 Representative transmission electron microscopy (TEM) images. We investigated endothelial niche, using TEM and were able to identify pericytes in both controls ( $\mathbf{a}$ and $\mathbf{b}$ ) and after $2 \mathrm{w}$ of bleomycin (c and $\mathbf{d}$ ). We did not find any lesser number of pericytes after bleomycin, and the pericytes appeared morphologically similar. Endothelial cells showed no apparent differences, but a slight edema was found after bleomycin, however no apoptotic endothelial cells were found (arrows).

lung parenchyma, and found a tendency toward increased expression $\quad(P=0.099)$ at $0.33 \mathrm{w} \quad\left(361 \pm 56 \quad\right.$ cells $\left./ \mathrm{mm}^{2}\right)$ compared with controls $\left(250 \pm 28\right.$ cells $\left./ \mathrm{mm}^{2}\right)$, whereas the expression was significantly decreased at later time points (Figure $7 \mathrm{a}$ and $\mathrm{c}$ ).

Wnt3a signaling is believed to initiate endothelial-tomesenchymal transition (EndMT), and vimentin has been described as a marker for EndMT. ${ }^{22}$ We therefore investigated the expression of vimentin in lungs and found increased levels at 1,3 , and $4 \mathrm{w}\left(68 \pm 14,96 \pm 14\right.$, and $93 \pm 11 \mathrm{cells} / \mathrm{mm}^{2}$, respectively) compared with controls $\left(57 \pm 7\right.$ cells $/ \mathrm{mm}^{2}$,
Figure $7 \mathrm{~d}$ and $\mathrm{f})$. A very strong tendency $(P=0.052)$ toward increased expression was found already at $0.33 \mathrm{w}(68 \pm 14$ cells $/ \mathrm{mm}^{2}$ ), suggesting that vimentin rapidly responded to bleomycin-induced tissue damage and stress, correlating relatively well with nuclear $\beta$-catenin positivity.

\section{Bleomycin Affects Small RhoGTPases}

RhoGTPases are crucial in processes requiring cytoskeletal rearrangement, including proliferation, contraction, and migration, as well as involved in Wnt signaling. A panRhoRacCDC42 marker showed that RhoGTPases were increased at $0.33 \mathrm{w}(1.72 \pm 0.16 \%$ of tissue area, $P=0.0064)$, $1 \mathrm{w}(5.29 \pm 0.99 \%, P<0.0001), 2 \mathrm{w}(1.64 \pm 1.04 \%, P=0.0182)$, $3 \mathrm{w} \quad(2.4 \pm 1.53 \%, \quad P=0.0008), \quad$ and $4 \mathrm{w} \quad(3.57 \pm 1.15 \%$, $P<0.0001)$ compared with controls $(0.49 \pm 0.29 \%)$.

In vitro experiments confirmed that both RhoA and Racl were increased (2 and 2.7-4.8 times, respectively) in microvascular endothelial cells following bleomycin administration (Figure $2 \mathrm{c}$ and $\mathrm{d}$ ). A migration assay showed no effect of bleomycin on the migration capacity of endothelial cells in vitro (data not shown).

\section{DISCUSSION}

We have previously described that repeated subcutaneous bleomycin administrations induced pulmonary fibrosis in parallel with inflammation, and how this process developed over time. ${ }^{6}$ It was however unknown how bleomycin may induce this effect. Our results here show that repeated subcutaneous bleomycin administrations affect the entire endothelial niche, activate Wnt signaling, and develop into fibrosis over time.

Bleomycin is used in high doses as an anticancer drug, exerting its effect by generating ROS, which diffuses freely over the cell membranes and causes single- and/or doublestranded DNA damage. ${ }^{23}$ When initiating pulmonary fibrosis with bleomycin, the most common administration route is intratracheal administration, which causes tissue necrosis, followed by an extensive inflammation and subsequent fibrosis. However, we have previously shown that repeated subcutaneous administration of a low-dose bleomycin results in a very different process, where fibrosis and inflammation develop in parallel resulting in increased proliferation rather than apoptosis. ${ }^{6}$

Within the present study, we found signs of oxidative stress (identified by Sod1) and DNA stress (p21 positivity) locally in lung, thus confirming the induction of oxidative stress by bleomycin in accordance with previous descriptions. ${ }^{2,3}$ Interestingly, Sod1 was normalized at 2 and $3 \mathrm{w}$, despite continuous bleomycin administrations. This may indicate the presence of other ROS-neutralizing enzymes, development of tolerance, or depletion of Sod1 protein. However, it is important to note that these time points ( 2 and $3 \mathrm{w}$ ) coincided with inflammation, as the numbers of neutrophils and macrophages peaked at these time points. This time shift pattern may indicate that inflammation itself elicited 


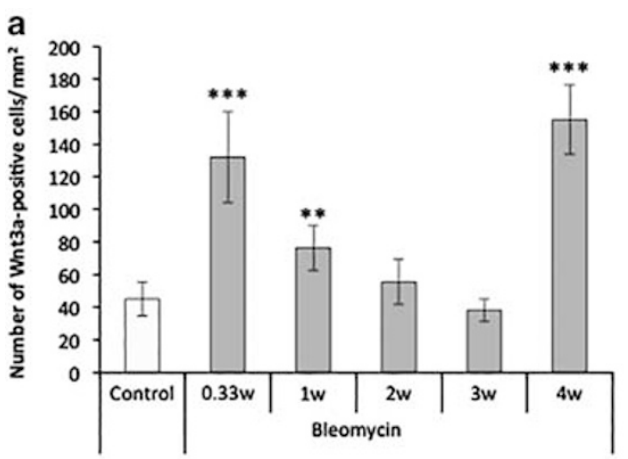

b
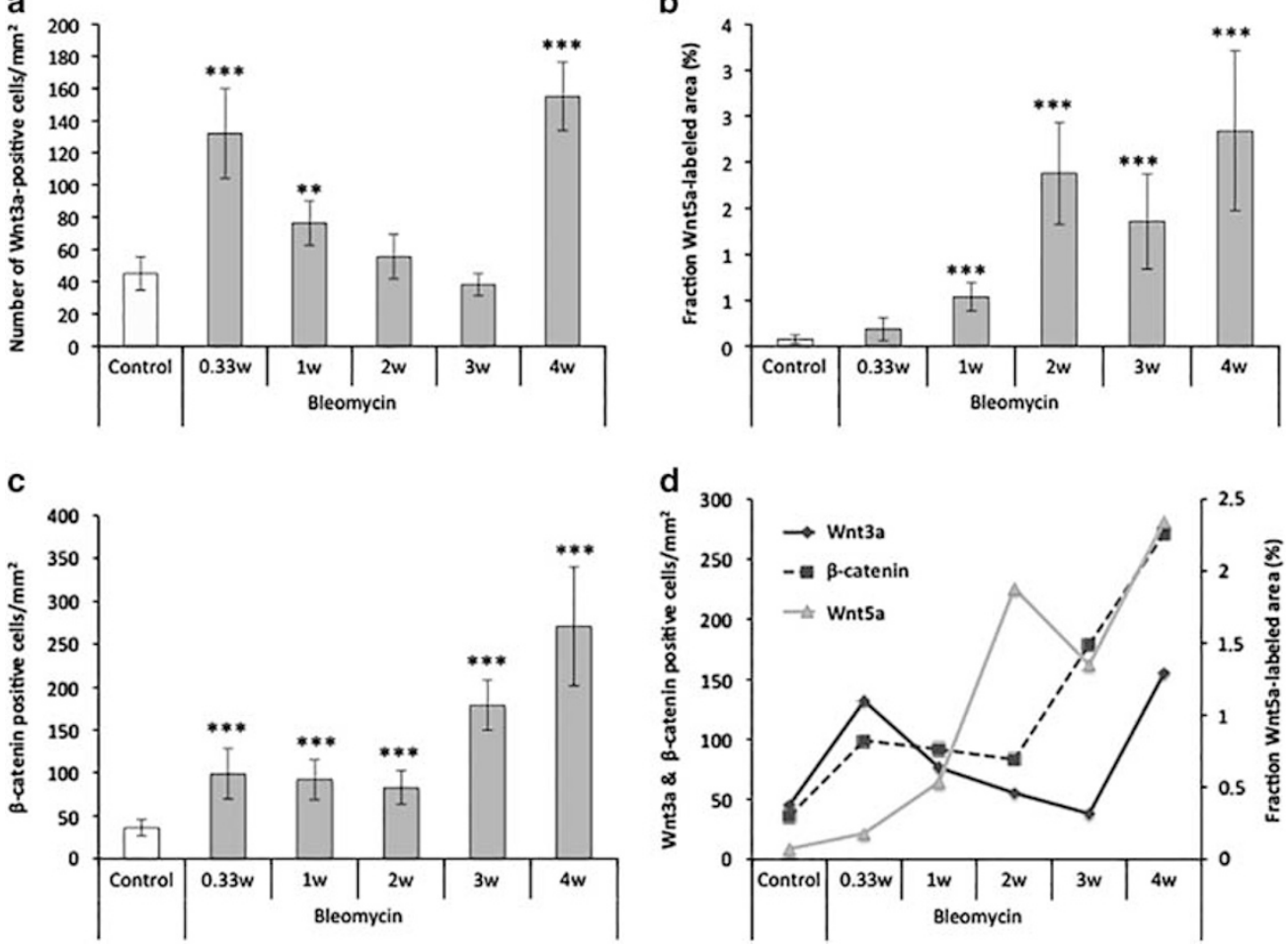

Figure 5 Canonical and non-canonical Wnt signaling during development of pulmonary fibrosis. We visualized Wnt3a (a) and Wnt5a (b) over time and found them to show an apparent temporal pattern, where Wnt3a increased rapidly following bleomycin, whereas Wnt5a responded slower. However, to quantify canonical signaling, we quantified the number of nuclei positive for $\beta$-catenin (c), and found these to be significantly increased at all times, peaking at 3 and $4 \mathrm{w}$. The expression pattern of Wnt3a, $\beta$-catenin and Wnt5a expression of Wnt3a, Wnt5a, and $\beta$-catenin over time can be found in (d). Data are given as either fraction (\%) labeled area of total tissue area (a), number of positively labeled cells $/ \mathrm{mm}^{2}(\mathbf{b})$, or nuclei $/ \mathrm{mm}^{2}(\mathbf{c})$ and given as mean \pm s.d. Bleomycin-treated groups were compared with controls using the Kruskal-Wallis test combined with LSD post hoc test, ${ }^{*} P<0.05,{ }^{* *} P<0.01$, and ${ }^{* * *} P<0.001$. LSD, least significant difference.

processes to minimize oxidative stress within the tissue, and these processes were also able to cope with the exogenous oxidative stress caused by bleomycin. A recent study highlights the proliferative effects and importance of ROS in wound healing and regeneration, showing that ROS is fundamental in responses to tissue damage. ${ }^{24}$ In accordance with this study, we found small RhoGTPases, crucial in cytoskeletal rearrangement, including proliferation ${ }^{14}$ to be increased, which we also found in our previous study. ${ }^{6}$ With the exception of Sod1, we were also able to confirm these increases in vitro, where microvascular endothelial cells were exposed to bleomycin. Sod1 was unaffected in vitro, which may suggest a fast response that has normalized at $24 \mathrm{~h}$ when the cells were harvested. This suggestion is supported by a study investigating Sod2 expression in response to bleomycin, found mRNA to peak at $6 \mathrm{~h}$ after addition of bleomycin, and normalized at $24 \mathrm{~h} .^{4}$ It is also likely that endothelial cells respond differently to oxidative stress in vitro, likely due to the absence of pericytes that are known to be important in the regulation of endothelial homeostasis.

From our previous work, we know that several types of structural cells within lung proliferate in response to subcutaneous bleomycin administrations, e.g., alveolar type 1-cells, fibroblasts, and endothelial cells. ${ }^{25}$ Due to close proximity, bleomycin can theoretically affect a large number of cell types directly via diffusing ROS, and we thus wanted to investigate whether bleomycin activates the endothelial cells specifically. To determine this, we investigated the expression of CD106/VCAM-1, which is a marker of activated endothelial cells. We found that the tissue expression of CD106 was increased and we thus conclude that endothelial cells are indeed activated by subcutaneous bleomycin administrations. Interestingly, the increased expression of CD106 correlated relatively well with the neutrophilia previously described within this model. ${ }^{6}$ This is further supported by an elegant study in vitro, where bleomycin exposure was found to increase the expression of CD106 on microvascular endothelial cells. ${ }^{26}$

As endothelial cells are activated by bleomycin administration it appears likely that also pericytes are affected, and we therefore investigated the number of NG2-positive cells. Unfortunately, there are no truly specific pericyte markers, but NG2 is a relatively specific marker for pericytes in the lung. The number of pericytes was significantly decreased at most time points, which was unexpected, as we found no obvious signs of endothelial dysfunction. It has been shown 

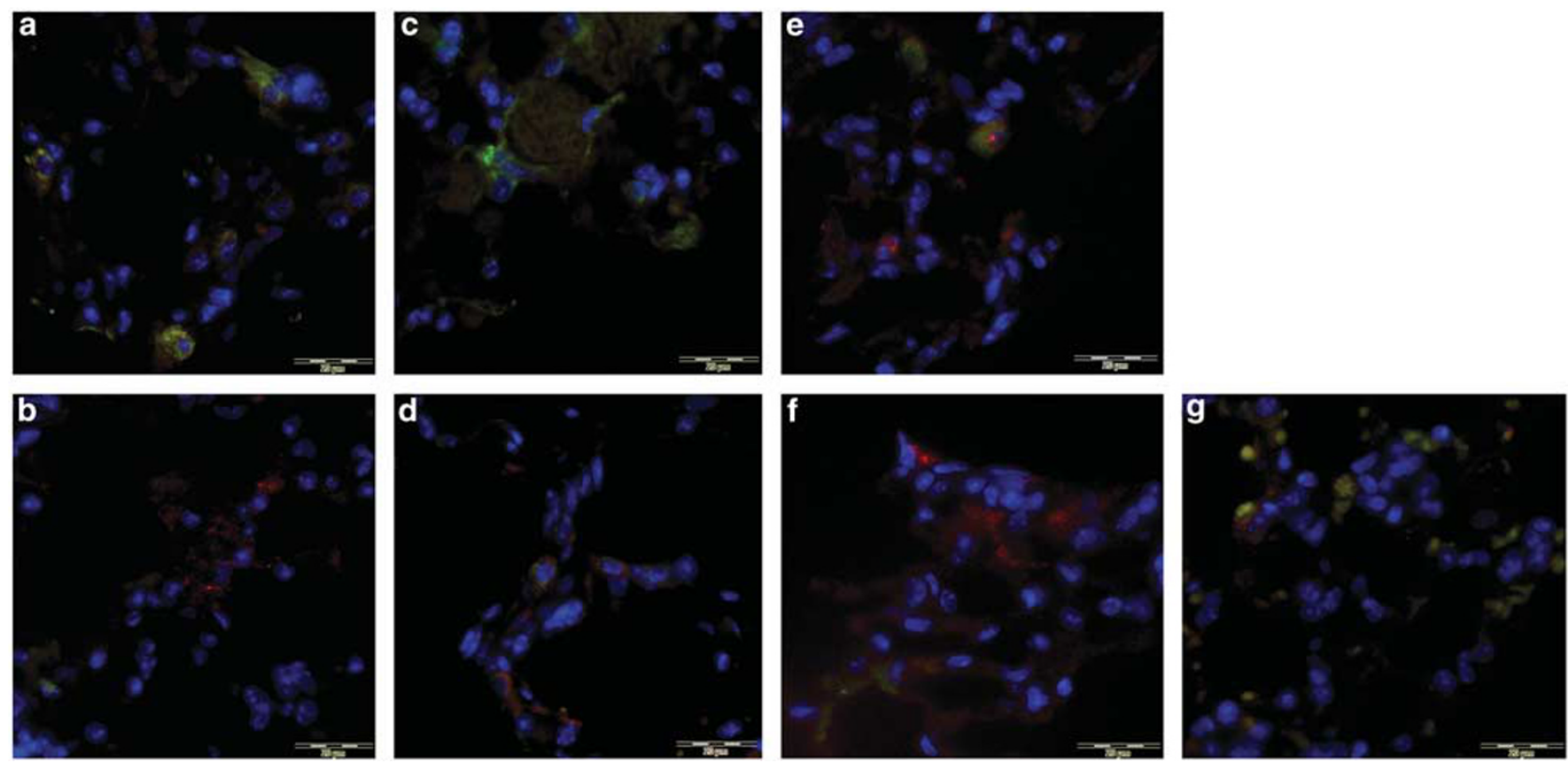

Figure 6 Expression and co-localization of NG2+Wnt3a, NG2+Wnt5a, and Wnt3a+Wnt5a at various time points. Pericytes (green) are weakly positive for Wnt3a (red) in healthy lung (a), but after one injection (0.33w) the number of pericytes has decreased and Wnt3a expression is increased (b). Wnt5a (red) is normally expressed at very low levels, and seldom by pericytes (green) in healthy lung (c) whereas after 4w of bleomycin increases total Wnt5a expression and some Wnt5a-positive pericytes can be seen (d). Double staining for Wnt3a (red) and Wnt5a (green) shows double-positive cells, as well as Wnt3a single-positive cells. Very few Wnt5a single-positive cells were found in normal lung (e). Following one bleomycin administration (0.33w), the expression of Wnt3a is increased, whereas cells rarely express Wnt5a (f). After $4 \mathrm{w}$ of bleomycin however, several cells co-positive for Wnt5a and Wnt3a are found (g). Nuclei are labeled with DAPI (blue). DAPI, 4',6-diamidino-2-phenylindole.

that inflammation may result in loss of microvascular pericyte coverage, ${ }^{27,28}$ however as the inflammatory response in our model was relatively modest, this appeared unlikely. Yet, to investigate pericytes closer, we studied tissue from controls and $2 \mathrm{w}$ (when inflammation peaks) using TEM. We were able to identify pericytes in both controls and after bleomycin administrations, with no apparent morphological differences. We thus conclude that the decreased NG2 staining most likely represented a decreased expression of NG2, rather than loss of cells.

The exact nature of pericytes is debated, but recent studies have highlighted their complex nature and importance. Pericytes were recently determined to exhibit trilineage differentiation properties and colony-forming activity similar to MSC. ${ }^{9}$ Intriguingly, a current study showed that perivascular MSC-like Gli1 ${ }^{+}$cells differentiate into myofibroblasts in response to tissue damage, and contribute to tissue fibrosis. ${ }^{20}$ These $\mathrm{Gli1}^{+}$cells have the ability to gain or loose the NG2 positivity suggesting them to be a differentiation state of pericytes. ${ }^{20}$ Together, these results suggest that pericytes are MSC-like cells in a specific differentiation state, and our results are in accordance with this elegant study. Taken together, others and our results indicate a key role of pericytes in the development of pulmonary fibrosis, but also entail problems such as how to study a cell type that dynamically changes surface markers, and how to define a pericyte.
Important regulators of progenitor and stem cells are the Wnt-signaling pathways, ${ }^{10}$ which are believed to keep these cells dedifferentiated and in a self-renewal state. ${ }^{11,12} \mathrm{Wnt}$ signaling is also important during wound healing where canonical signaling stabilizes the $\beta$-catenin complex within the cytosol and relocalizes this complex to the nucleus, resulting in gene transcription. Target genes of $\beta$-catenin include genes involved in tissue repair, and regeneration such as cyclinD1, CD44, and VEGF. ${ }^{13}$ Canonical Wnt signaling is normally strictly regulated, and one control mechanism is non-canonical signaling (induced by, e.g., Wnt5a), which inhibits gene expression. ${ }^{29}$ The involvement of Wnt signaling in the development of fibrosis can therefore be expected by several reasons, and has previously been described ${ }^{17,18,30}$ and reviewed. ${ }^{31}$ Interestingly, the number of cells positive for Wnt $3 \mathrm{a}$ and $\beta$-catenin did not correlate completely, as Wnt3a was normalized at $3 \mathrm{w}$, whereas $\beta$-catenin was increased, which may indicate an effect of other Wnt:s, not investigated within this study. A complete mapping of the Wnt signaling was unfortunately beyond the scope of this study due to the highly complex nature of this signaling pathway; two main transduction pathways (canonical and non-canonical), two different non-canonical pathways (Wnt/PCP and $\mathrm{Wnt} / \mathrm{Ca}^{2+}$ ), several Wnt ligands and receptors whose interactions are not yet fully mapped as well as complicated regulatory mechanisms. 
a
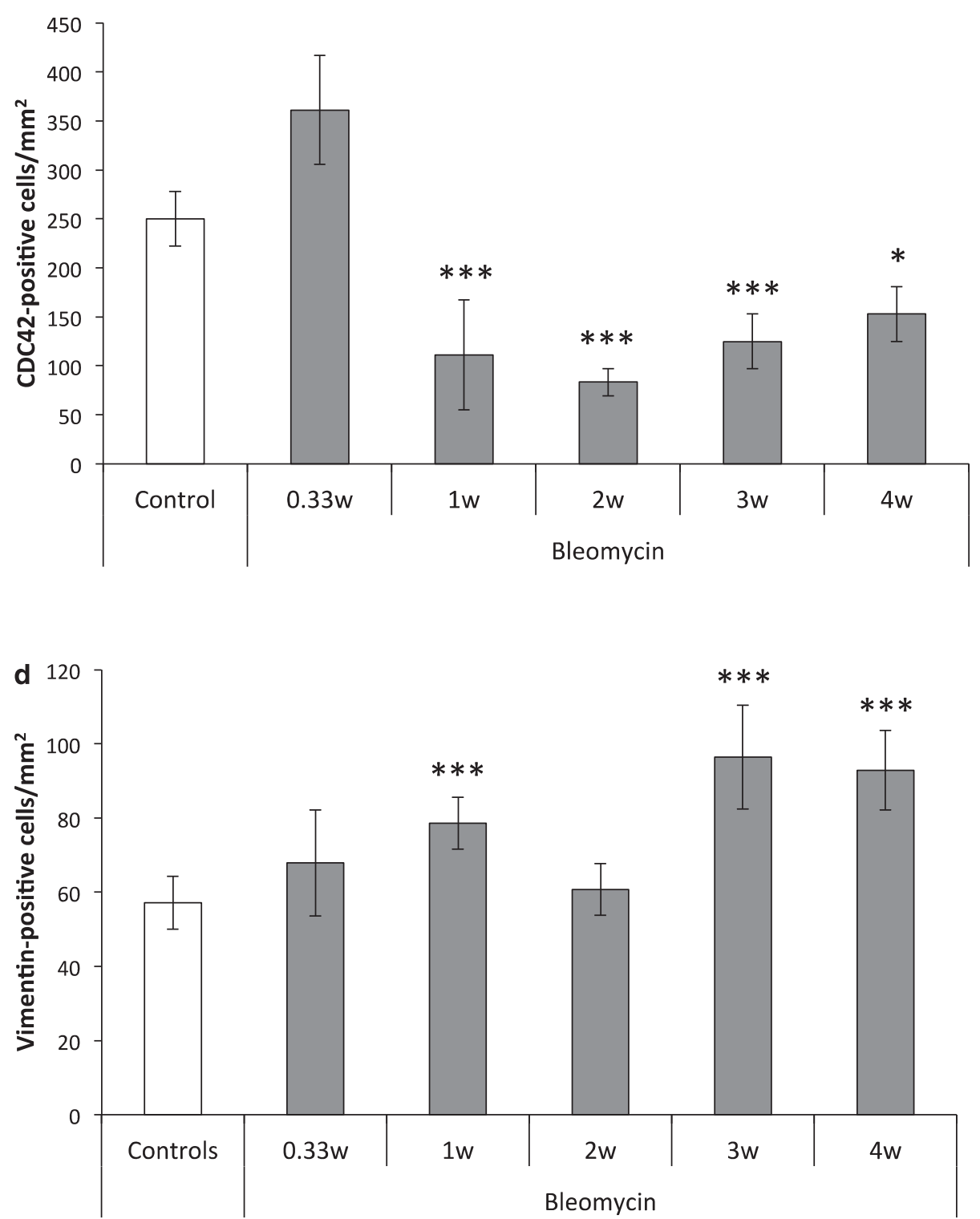
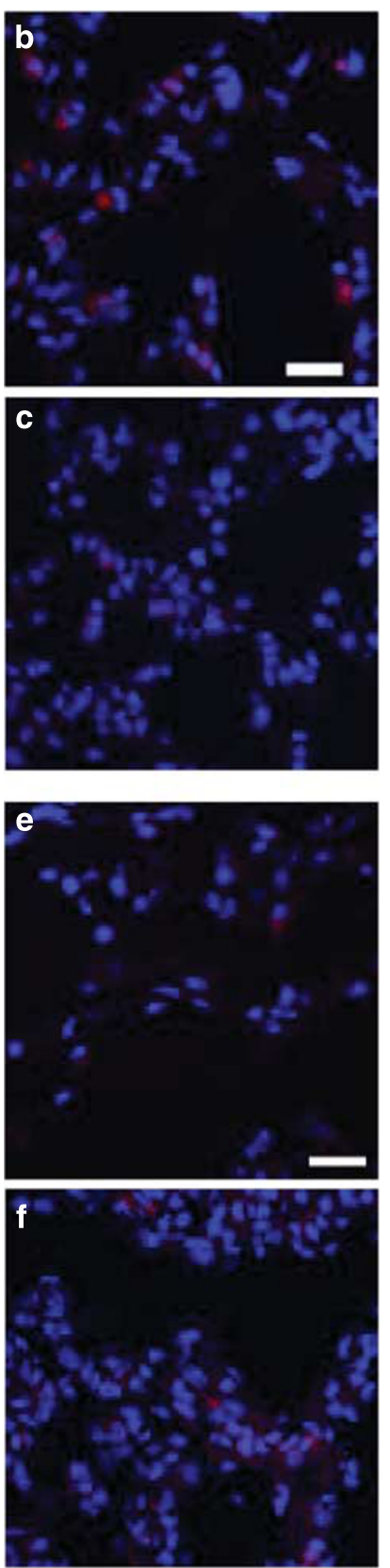

Figure 7 Effects of bleomycin administration on CDC42 and vimentin. CDC42 has been described to mediate a switch from canonical to non-canonical signaling. We therefore investigated the expression of CDC42 (a) in tissue (red staining) and found a tendency toward increased positivity at $0.33 \mathrm{w}$ (when canonical signaling was increased) compared with controls (b) but from $1 \mathrm{w}$ the number of CDC42-positive cells was significantly decreased, with the lowest numbers at $2 \mathrm{w}$ (c). Vimentin has among other things been described as a marker of endothelial-to-mesenchymal transition (EndMT) and EndMT genes are among genes induced by canonical signaling. We therefore investigated the number of vimentin-positive cells (d, red staining) at different time points, and found vimentin to be increased compared with controls (e) at 1, 3 (f), and $4 \mathrm{w}$. Data are either given as number of positively labeled cells $/ \mathrm{mm}^{2}$ or given as mean \pm s.d. Scale bar represents $20 \mu \mathrm{m}$ in all images. Bleomycin-treated groups were compared with controls using the Kruskal-Wallis test combined with LSD post hoc test, ${ }^{*} P<0.05$, ${ }^{* *} P<0.01$, and ${ }^{* * *} P<0.001$. LSD, least significant difference.

Wnt3a signaling is believed to initiate EndMT, and vimentin has been described as a marker for EndMT. ${ }^{22}$ Furthermore, we identified $\alpha$-SMA-positive endothelial cells, which is indicative of EndMT. However, pericytes within the blood-brain barrier are known to express high amounts of vimentin, ${ }^{32}$ and it is possible that the increased vimentin expression within our study partly represents an increase in the number or activity of pericytes rather than EndMT. Our data thus suggest that both EndMT and pericyte-tomesenchymal transition occurs within this model.

In contrast to Wnt3a, which was relatively abundant in healthy lung, Wnt5a expression was found to be very low in 
healthy lung. We found Wnt3a to increase rapidly in response to bleomycin, whereas Wnt5a increased more slowly. This likely indicates a temporal shift in Wnt3a and Wnt5a expression, which may be interpreted as Wnt5a exerting control over canonical signaling. The complex interplay between canonical and non-canonical signaling clearly warrants further investigation.

We could histologically confirm a low expression of Wnt3a but not Wnt5a in pericytes in healthy lungs. After bleomycin, however, most NG2-positive cells were also expressing Wnt5a, whereas few NG2-positive cells expressed Wnt3a. These results may suggest that increased Wnt $3 \mathrm{a}$ is associated with the loss of NG2-expression, although it is currently unknown whether Wnt3a is a cause of, or a result from, decreased NG2 expression.

A recent study described the switch from canonical to non-canonical Wnt signaling in HSC, and pinpointed a role of the small Rho GTPase CDC42 mediating the transition. ${ }^{21}$ Our results show that the tissue expression of CDC42 is significantly decreased from $1 \mathrm{w}$ when Wnt5a is significantly increased, which may suggest a similar switch being present within the lung parenchyma.

When investigating alterations over time, we detected a 'normalization' at $3 \mathrm{w}$, which was lost at $4 \mathrm{w}$, when most parameters were significantly increased compared with controls. This may suggest a biphasic development, and based on the results within this study, we believe this may be due to a collapse in regenerative control mechanisms, as this coincided with a marked increase in canonical signaling (increased number of $\beta$-catenin-positive nuclei). It appears that a shift occurs around the 3rd week, from an acute but normal wound repair, into a more chronic process.

By using a histological approach we were able to specifically investigate a defined lung compartment, the parenchyma. The interactions between cells within this compartment are of utmost importance, and it is likely that cellular communication is a key process in the development of pulmonary fibrosis. Based on our results, we conclude that bleomycininduced oxidative stress causes mild tissue damage and DNA stress, affecting the entire endothelial niche. The tissue damage activates repair processes involving canonical and non-canonical Wnt-signaling and due to the repeated exposures, disrupts a normally fine-tuned Wnt balance, which causes MSC-like pericytes to alter phenotype. As pericytes may differentiate into myofibroblasts, understanding the effects of Wnt signaling in the endothelial niche now emerges as an important objective to understand the early events of pulmonary fibrosis.

Supplementary Information accompanies the paper on the Laboratory Investigation website (http://www.laboratoryinvestigation.org)

\section{ACKNOWLEDGMENTS}

We acknowledge the skilled help of Kristina Lexmüller. Funded by grants from the Swedish Research Counsel, the Swedish Heart-Lung Foundation, the
Gyllenstierna Krapperup's Foundation, the Royal Physiographic Society, the Sandberg Foundation and the Faculty of Medicine, Lund University.

\section{DISCLOSURE/CONFLICT OF INTEREST}

The authors declare no conflict of interest.

1. Borensztajn K, Crestani B, Kolb M. Idiopathic pulmonary fibrosis: from epithelial injury to biomarkers-insights from the bench side. Respiration 2013;86:441-452.

2. Gadjeva VG, Nikolova GD, Grigorov BG et al. Spin-labelled 1-ethyl-1nitrosourea prevents doxorubicin and bleomycin-induced oxidative stress in lungs, hearts and kidneys of tumour-bearing mice. Adv Biol Chem 2014;4:291-300.

3. El-Khouly D, El-Bakly WM, Awad AS et al. Thymoquinone blocks lung injury and fibrosis by attenuating bleomycin-induced oxidative stress and activation of nuclear factor kappa-B in rats. Toxicology 2012;302: 106-113.

4. Kokot A, Sindrilaru A, Schiller $M$ et al. Alpha-melanocyte-stimulating hormone suppresses bleomycin-induced collagen synthesis and reduces tissue fibrosis in a mouse model of scleroderma: melanocortin peptides as a novel treatment strategy for scleroderma? Arthritis Rheum 2009;60:592-603.

5. Wallach-Dayan SB, lzbicki G, Cohen PY et al. Bleomycin initiates apoptosis of lung epithelial cells by ros but not by Fas/Fasl pathway. Am J Physiol Lung Cell Mol Physiol 2006;290:L790-L796.

6. Rydell-Tormanen K, Andreasson K, Hesselstrand R et al. Extracellular matrix alterations and acute inflammation; developing in parallel during early induction of pulmonary fibrosis. Lab Invest 2012;92: 917-925.

7. Fasske $E$, Morgenroth $\mathrm{K}$. Experimental bleomycin lung in mice. A contribution to the pathogenesis of pulmonary fibrosis. Lung 1983;161:133-146.

8. Armulik A, Genove G, Betsholtz C. Pericytes: developmental, physiological, and pathological perspectives, problems, and promises. Dev Cell 2011;21:193-215.

9. Rowley JE, Johnson JR. Pericytes in chronic lung disease. Int Arch Allergy Immunol 2014;164:178-188.

10. Katoh $M$, Katoh $M$. Wnt signaling pathway and stem cell signaling network. Clin Cancer Res 2007;13:4042-4045.

11. Holland JD, Klaus A, Garratt AN et al. Wnt signaling in stem and cancer stem cells. Curr Opin Cell Biol 2013;25:254-264.

12. Nusse R. Wnt signaling and stem cell control. Cell Res 2008;18:523-527.

13. Baarsma HA, Konigshoff M, Gosens R. The Wnt signaling pathway from ligand secretion to gene transcription: molecular mechanisms and pharmacological targets. Pharmacol Ther 2013;138:66-83.

14. Schlessinger $\mathrm{K}$, Hall $\mathrm{A}$, Tolwinski N. Wnt signaling pathways meet Rho Gtpases. Genes Dev 2009;23:265-277.

15. De Langhe SP, Reynolds SD. Wnt signaling in lung organogenesis. Organogenesis 2008;4:100-108.

16. Carthy JM, Garmaroudi FS, Luo Z et al. Wnt3a induces myofibroblast differentiation by upregulating Tgf-Beta signaling through Smad2 in a beta-catenin-dependent manner. PLoS One 2011;6:e19809.

17. Vuga $\amalg$, Ben-Yehudah A, Kovkarova-Naumovski $E$ et al. Wnt5a is a regulator of fibroblast proliferation and resistance to apoptosis. Am J Respir Cell Mol Biol 2009;41:583-589.

18. Konigshoff M, Balsara N, Pfaff EM et al. Functional Wnt signaling is increased in idiopathic pulmonary fibrosis. PLoS One 2008;3:e2142.

19. Rydell-Tormanen $\mathrm{K}$, Westergren-Thorsson $\mathrm{G}$. Wnt-imbalance within the endothelial niche contributes to pulmonary fibrosis [Abstract]. International Colloquium on Lung and Airway Fibrosis 2014;Vol. 18, MontTremblant, $\mathrm{p} 20$.

20. Kramann R, Schneider RK, DiRocco DP et al. Perivascular Gli1(+) progenitors are key contributors to injury-induced organ fibrosis. Cell Stem Cell 2015;16:51-66.

21. Florian MC, Nattamai KJ, Dorr K et al. A canonical to non-canonical Wnt signalling switch in haematopoietic stem-cell ageing. Nature 2013;503: 392-396.

22. Ivaska J. Vimentin: central hub in Emt induction? Small GTPases 2011;2: 51-53.

23. Chen J, Stubbe J. Bleomycins: Towards better therapeutics. Nat Rev Cancer 2005;5:102-112. 
24. Gauron C, Rampon C, Bouzaffour M et al. Sustained production of Ros triggers compensatory proliferation and is required for regeneration to proceed. Sci Rep 2013;3:2084.

25. Rydell-Tormanen K, Andreasson K, Hesselstrand R et al. Absence of fibromodulin affects matrix composition, collagen deposition and cell turnover in healthy and fibrotic lung parenchyma. Sci Rep 2014;4:6383.

26. Williamson L, Sadofsky J, Greenman $\mathrm{S}$ et al. A potential role for endothelial adhesion molecules and cytokines in chemotherapyinduced lung fibrosis [Abstract]. ERS International Congress Munich 2014, p 3841.

27. Johnson JR, Folestad E, Rowley JE et al. Pericytes contribute to airway remodeling in a mouse model of chronic allergic asthma. Am J Physiol Lung Cell Mol Physiol 2015;308:L658-L671.
28. Fuxe J, Tabruyn $\mathrm{S}$, Colton $\mathrm{K}$ et al. Pericyte requirement for anti-leak action of angiopoietin-1 and vascular remodeling in sustained inflammation. Am J Pathol 2011;178:2897-2909.

29. Smit $L$, Baas A, Kuipers J et al. Wnt activates the Tak1/Nemo-like kinase pathway. J Biol Chem 2004;279:17232-17240.

30. Aumiller V, Balsara N, Wilhelm J et al. Wnt/Beta-catenin signaling induces II-1 beta expression by alveolar epithelial cells in pulmonary fibrosis. Am J Respir Cell Mol Biol 2013;49:96-104.

31. Konigshoff $\mathrm{M}$, Eickelberg $\mathrm{O}$. Wnt signaling in lung disease: a failure or a regeneration signal? Am J Respir Cell Mol Biol 2010;42:21-31.

32. Bandopadhyay R, Orte C, Lawrenson JG et al. Contractile proteins in pericytes at the blood-brain and blood-retinal barriers. J Neurocytol 2001;30:35-44 